\title{
Laparotomía exploratoria por abdomen agudo durante terapia de oxigenación por membrana extracorpórea, reporte de caso
}

\section{Exploratory laparotomy for acute abdomen during extracorporeal membrane oxygenation therapy, case report}

\author{
Nancy V. Alva-Arroyo ${ }^{1 *}$, Ibzan J. Salvador-Ibarra ${ }^{1}$, José C. Gasca-Aldama ${ }^{2}$ y Gabriela Rodríguez-Ruíz ${ }^{3}$ \\ ${ }^{1}$ Unidad de Terapia Intensiva, Hospital Ángeles Mocel, Universidad Nacional Autónoma de México; ${ }^{2}$ Unidad de Terapia Intensiva, Hospital Juárez de \\ México, Universidad Nacional Autónoma de México; ${ }^{3}$ Servicio de Cirugía General, Hospital San Ángel Inn Patriotismo. Ciudad de México, México
}

\section{Introducción}

La terapia de oxigenación por membrana extracorpórea (ECMO, extracorporeal membrane oxygenation) puede ser un factor de riesgo adicional de complicaciones abdominales, volviéndose una necesidad la laparatomía de emergencia ${ }^{1}$. Los pacientes sometidos a procedimientos quirúrgicos durante la ECMO incrementan el riesgo de complicaciones hemorrágicas atribuibles a la anticoagulación ${ }^{2}$. Además de la anticoagulación, las alteraciones fisiológicas, que comprenden alteraciones ácido-base, inestabilidad hemodinámica y coagulopatía, colocan a estos pacientes en un mayor riesgo de complicaciones quirúrgicas ${ }^{2}$.

Desde 1980, La gastrostomia endoscopica percutanea, ha desplazado prácticamente a la gastrostomía quirúrgica ${ }^{3}$. Las complicaciones tras la realización de una gastrostomía endoscópica percutánea son escasas; su incidencia es $<17 \%$, y las graves son $<3 \%$. La hemorragia abdominal puede aparecer de forma inmediata por punción de un vaso gástrico; la hemorragia masiva está reportada en varias series hasta en un $2.5 \%$ de los casos. La peritonitis aguda es una complicación grave, asociada con alta mortalidad, y se produce por fuga del contenido gástrico antes del trayecto fistuloso, por tracción o arrancamiento de la sonda ${ }^{3}$.

Larsson et al. ${ }^{4}$ muestran que cuando está completamente desarrollada, la coagulopatía es muy difícil de revertir. En este estudio muestra que la ECMO puede mejorar la acidosis y la temperatura en un modelo hemorrágico experimental traumático ${ }^{4}$, Renaudier et al demostraron que la isquemia mesenterica aguda es relativamente frecuente (OR)4.5, (IC) 95\%, $(p=0.02)^{5}$.

La trombosis es una de las complicaciones más frecuentes y temidas del soporte con ECMO. Se desconoce la verdadera incidencia de complicaciones tromboembólicas, pero los estudios de autopsia sugerieren que la evaluación clínica subestima su aparición.

En el informe anual más reciente de la Extracorporeal Life Support Organization (ELSO) se informó la aparición de coágulos en el oxigenador en casi el 13\% de los pacientes. Los coágulos adicionales en otras partes del circuito eran más comunes en los pacientes con ECMO para problemas cardíacos que en los de apoyo respiratorio ${ }^{6,7}$.

El sangrado, por el contrario, es siempre in vivo y puede ocurrir en los sitios de inserción de la cánula o del catéter, recientes incisiones quirúrgicas, nariz, boca, tracto urinario, cavidad abdominal o torácica,

\section{Correspondencia:}

*Nancy V. Alva-Arroyo

E-mail: nancy.alva.ar@anahuac.mx
Fecha de recepción: 30-11-2021

Fecha de aceptación: 17-12-2021 DOI: 10.24875/REIE.21000215
Disponible en internet: 07-03-2022

Rev Educ Investig Emer. 2022;4(Supl 1):99-101 www.medicinadeemergencias.com bajo la licencia CC BY-NC-ND (http://creativecommons.org/licenses/by-nc-nd/4.0/). 
pulmones y vías respiratorias, o gastrointestinal. La etiología del sangrado es multifactorial. La presión arterial alta o el $\mathrm{CO}_{2}$ bajo están asociados con hemorragia intracraneal. La gastritis y la úlcera péptica pueden causar sangrado gastrointestinal. Las etiologías hematológicas incluyen, entre otras, anticoagulación, deficiencia de coagulación, factores que incluyen el factor XIII, trombocitopenia, defectos de la función plaquetaria, hiperfibrinólisis y sustancias similares a la heparina. En el interior de los vasos, la hemólisis puede contribuir tanto al sangrado como a la formación de coágulos ${ }^{8}$.

La incidencia de complicaciones relacionadas con la ECMO, según Chung et al. ${ }^{9}$ en su estudio, incluyen reexploración por sangrado (19/50,38\%), infección documentada por hemocultivos $(4 / 50,8 \%)$, trombosis $(7 / 50,14 \%)$, isquemia de extremidades inferiores $(7 / 59$, $14 \%$ ), hemólisis y requerimiento de plasma fresco $(2 / 50,4 \%)$, fuga del oxigenador $(13 / 50,26 \%)$, cambio de sistema de ECMO (9/50, 18\%), falla renal (9/50, $18 \%)$, disfunción hepática $(5 / 50,10 \%)$, disfunción neurológica $(3 / 50,6 \%)$, coagulopatía intravascular diseminada $(1 / 50,2 \%)$ y sangrado gastrointestinal $(3 / 50,6 \%)$.

\section{Caso clínico}

Varón de 29 años, sin antecedentes de importancia, que ingresó por neumonía debida a COVID-19, con disnea y desaturación, siendo su día 6 de contagio. Ingresó a piso de hospitalización, con puntas de alto flujo y en posición prono, e índice ROX 4.9, por lo que se mantuvo por 48 horas; sin embargo, mostró fiebre de $38.5^{\circ} \mathrm{C} \mathrm{e}$ índice ROX 2.4 con indicación de fracaso al alto flujo, por lo que fue llevado a terapia intensiva, con frecuencia respiratoria (FR) 45 y saturación por pulsioximetría del $80 \%$. Fue intubado y se inició sedación profunda guiada por índice biespectral 40, relajación neuromuscular, infusión continua, medidas de protección alveolar y posición prono. Mostró una discreta mejoría en las primeras 48 horas de intubación, pero el día 3 presentaba un índice presión arterial de oxígeno/fracción inspirada de oxígeno $\left(\mathrm{PaO}_{2} / \mathrm{FiO}_{2}\right)$ de $89 \mathrm{mmHg}$, sacrificando la concentración arterial de oxígeno $65 \mathrm{mmHg}$ para hipercapnia permisiva y con esto lograr metas de volumen tidal y driving pressure, sin respuesta, saturando al $79 \%$ con $\mathrm{FiO}_{2}$ del $100 \%$, incrementando presiones, motivo por el cual se decide iniciar ECMO a los 6 días de hospitalización y 4 días de intubación.

Fue valorado por el equipo de ECMO, siendo candidato de acuerdo con los criterios de la ELSO, el NHS Cesar Trial, el Eolia Trial y la Sociedad Chilena de

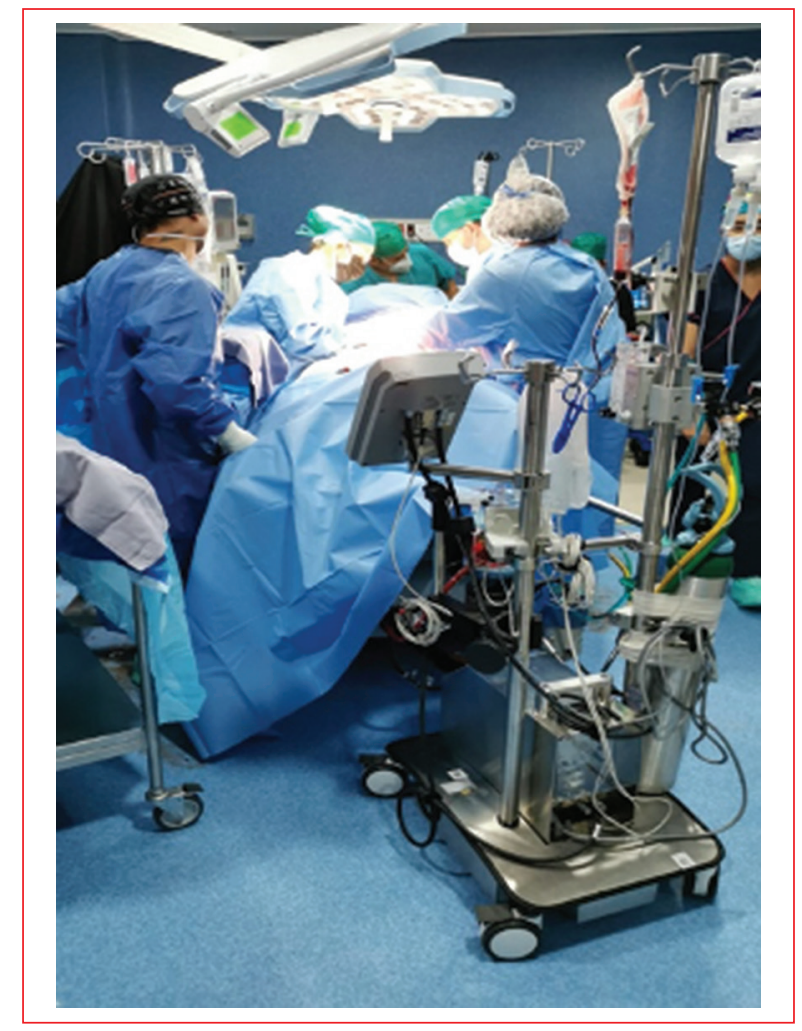

Figura 1. Primera laparotomía exploratoria por choque hemorrágico. durante el traslado y transoperatorio, soporte extracorporeo ininterrumpido.

Medicina Intensiva. La duración de la ECMO fue de 35 días. Debido a la estancia prolongada, se decidió realizar traqueotomía y posteriormente gastrostomía percutánea endoscópica para alimentación, la cual se realizó a pie de cama durante la mañana; sin embargo, evolucionó inmediatamente con datos de choque hipovolémico, presión arterial de $70 / 30 \mathrm{mmHg}$, frecuencia cardiaca de 145 latidos por minuto, gesticulando a la palpación abdominal, pálido, con mayor disminución de las cifras tensionales, abdomen agudo, ultrasonido positivo y sangrado en correderas, por lo que ingresó a quirófano de urgencias. Se realizó la primera laparotomía exploratoria (Fig. 1), con los siguientes hallazgos: sangrado de la arteria gastroepiploica, aproximadamente $2500 \mathrm{ml}$ en la cavidad (libre y coágulos), con mejoría clínica al cohibir la hemorragia. A los 5 días de postoperatorio, nuevamente dolor en el abdomen, rebote positivo, drenajes abdominales con salida de material de nutrición, ingreso a quirófano y segunda laparotomía exploratoria (Fig. 2), encontrando salida de la sonda de gastrostomía, que se reparó y se cambió a yeyunostomía; reingreso a terapia intensiva con mejoría de las constantes vitales. Actualmente el paciente se encuentra iniciando jornadas laborales en casa. 


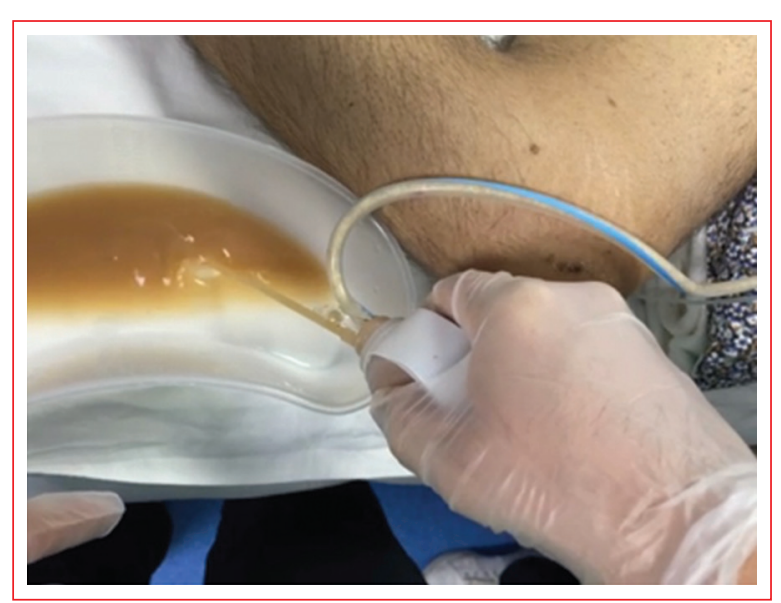

Figura 2. Segunda laparotomía exploratoria. Se observa material gastroalimentario por drenajes abdominales (peritonitis aguda) debido a salida de la sonda de gastrostomía a la cavidad peritoneal.

Se cuenta con permiso, asentado en el consentimiento informado por parte del paciente, para exponer el caso y las fotografías con fines académicos, y se han seguido las recomendaciones de las guías CARE para reporte de casos.

\section{Conclusiones}

Durante la ECMO, el sangrado y la trombosis son problemas comunes. La patología intraabdominal aguda que requiere laparotomía en los pacientes con ECMO resulta compleja debido a diferentes factores. Nosotros tratamos con éxito un caso de perforación de estómago por colocación de sonda de gastrostomía endoscópica, y posteriormente peritonitis por salida de la sonda de gastrostomía ocasionando vaciamiento del contenido gástrico y alimenticio a la cavidad abdominal. Estos dos eventos abdominales se identificaron inmediatamente y se realizó en dos ocasiones laparotomía exploratoria, con una diferencia de 5 días entre ellas, siendo abordadas con éxito. Sin embargo, la isquemia mesentérica aguda es una complicación relativamente frecuente y dramática entre los pacientes con ECMO. Además, las complicaciones hemorrágicas y trombóticas siguen siendo unas de las principales causas de morbilidad y mortalidad en los pacientes con ECMO.

Lidiar con hemorragias y trombosis es un problema actual para los pacientes con ECMO. Las etiologías de la hemorragia y de la trombosis son multifactoriales e incluyen, entre otras, deficiencia de factor de coagulación de consumo, activación excesiva de la fibrinólisis, trombocitopenia y disfunción plaquetaria. La hemólisis intravascular también desempeña un papel importante para el trastorno hemostático. Se requiere seguimiento continuo y estricto para identificar de manera oportuna estas complicaciones y no llevar al deterioro o a un desenlace fatal.

\section{Financiamiento}

Los autores no recibieron financiamiento para llevar a cabo este artículo.

\section{Conflicto de intereses}

Los autores declaran no tener conflictos de intereses.

\section{Responsabilidades éticas}

Protección de personas y animales. Los autores declaran que para esta investigación no se han realizado experimentos en seres humanos ni en animales.

Confidencialidad de los datos. Los autores declaran que han seguido los protocolos de su centro de trabajo sobre la publicación de datos de pacientes.

Derecho a la privacidad y consentimiento informado. Los autores han obtenido el consentimiento informado de los pacientes y/o sujetos referidos en el artículo. Este documento obra en poder del autor de correspondencia

\section{Bibliografía}

1. McCann C, Adams K, Schizas A, George M, Barrett N, Wyncoll D, et al. Outcomes of emergency laparotomy in patients on extracorporeal membrane oxygenation for severe respiratory failure: a retrospective, observational cohort study. J Crit Care. 2019;53:253-7.

2. Wesley E, Castle S, Golden J, Rosenberg D, Jensen A, Bliss D. Laparotomy complications on extracorporeal life support: surgical site bleeding does not increase mortality. J Pediatr Surg. 2019;54:1736-9.

3. Domingo J. Gastrostomía endoscópica percutánea. Actualizaciones temáticas Madaus en gastroenterología. Servicio de Aparato Digestivo. Zaragoza: Hospital Universitario Miguel Servet; 2000. p. 1-15.

4. Larsson M, Forsman P, Hedenqvist P, Öustlund A, Hultman J, Wilkman A, et al. Extracorporeal membrane oxygenation improves coagulopathy in an experimental traumatic hemorrhagic model. Eur J Trauma Emerg Surg. 2017;43:701-9.

5. Renaudier M, Roux Q, Bougouin W, Boccara J, Attias A, Fiore A, et al. Acute mesenteric ischaemia in refractory shock on veno-arterial extracorporeal membrane oxygenation. Eur Heart J Acute Cardiovasc Care. 2021;10:1-9.

6. Periche E, Koborzan R, Sbraga F, Blasco L. Outcomes of extracorporeal membrane oxigenation in adult patients with hypoxemic respiratory failure refractory to mechanical ventilation. Respir Med Case Rep. 2018;25:220-4.

7. Murphy D, Hockings L, Andrews R, Aubron C, Gardiner E, Pellegrino V. Extracorporeal membrane oxygenation - hemostatic complications. Transfus Med Rev. 2015;5:90-101.

8. Thomas J, Kostousov V, Teruya J. Bleending and trombotic complications in the use of extracorporeal membrane oxygenation. Semin Thromb Hemost. 2018;44:20-9.

9. Chung M, Zhao YY, Strom J, Changyu S, Yeh R. Extracorporeal membrane oxygentation use in cardiogenic shock. Impact age on in-hospital mortality, length of stay and costs. Crit Care Med. 2019;47:214-21. 